\title{
Modified pedicle screw-rod versus anterior subcutaneous internal pelvic fixation for unstable pelvic anterior ring fracture: A retrospective study and finite element analysis
}

\section{Sheng-Long Ding}

Qingpu Branch of Zhongshan Hospital,Fundan University https://orcid.org/0000-0003-0682-3596

Fan-Cheng Chen

Zhongshan Hospital,Fundan University

Jun-Ming Huang

Zhongshan Hospital,Fudan University

\section{Guang-Ming Zhang}

Qingpu Branch of Zhonsghan Hospital,Fudan University

\section{Fu-Yong Wang}

Qingpu Branch of Zhongshan Hospital,Fudan University

Jing-Hui Lei

Qingpu Branch of Zhongshan Hospital,Fudan University

\section{Xiao-Tian Wu}

Qingpu Branch of Zhongshan Hospital,Fudan University

Zhi-yong Ruan ( $\nabla$ xyrzy818@163.com )

\section{Research article}

Keywords: Pelvic fracture, Anterior ring, High-energy trauma, Minimally invasive fixation

Posted Date: July 22nd, 2020

DOl: https://doi.org/10.21203/rs.3.rs-16488/v2

License: (c) (1) This work is licensed under a Creative Commons Attribution 4.0 International License. Read Full License 


\section{Abstract}

Objectives: This study compared the stability and clinical outcomes of modified pedicle screw-rod fixation (MPSRF) and anterior subcutaneous internal pelvic fixation (INFIX) for the treatment of anterior pelvic ring fractures using Tornetta and Matta grading system and finite element analyses.

Methods: In a retrospective review of a consecutive patient series, 63 patients with Orthopaedic Trauma Association (OTA)/Arbeitsgemeinschaft für Osteosynthesefragen (AO) type $\mathrm{B}$ or $\mathrm{C}$ pelvic ring fractures were treated by MPRSF $(n=30)$ or INFIX $(n=33)$. The main outcome measure were Majeed score, incidence of complications and adverse outcomes, and fixation stability was evaluated by finite element analysis (FEA).

Results: two groups did not differ in terms of injury severity score, OTA classification, cause of injury, and time to pelvic surgery. However, the MPSRF group had a higher satisfactory rate according to the Tornetta and Matta grading system than the INFIX group (73.33\% vs $63.63 \%)$ as well as a higher Majeed score (81.5 \pm 10.4 vs $76.3 \pm 11.2$ ), which was statistically significant at 6 months' post-surgery. FEA showed that MPRSF was stiffer and more stable than INFIX and had a lower risk of implant failure.

Conclusions: Both MPSRF and INFIX have acceptable biomechanical stability for the treatment of unstable pelvic anterior ring fractures. However, MPRSF has better fixation stability and lower risk of implant failure, which can lead to better clinical outcomes.

\section{Introduction}

Anterior pelvic structures are more fragile and prone to fracture than dorsal parts as they bear higher loads [1]. Clinically, high-energy pelvic ring fractures leading to decreased functionality account for approximately $1.5 \%-3.9 \%$ of all fractures [2]. The high rates of morbidity and mortality are of concern to orthopedists and an economic burden to patients and society.

External fixation devices and ORIF are the primary modality of treatment for extremity and pelvic trauma, which could provide a rapid and stiff fixed strength. [3]. However, it is associated with complications such as pin tract infection (in $2 \%-50 \%$ ), fixator loosening (in $0 \%-20 \%$ ), loss of reduction $(0 \%-30 \%$ ), and restriction of daily activities, particularly in obese patients $[4,5]$. Moreover, open reduction has the potential disadvantage of extensive exposure including muscle stripping, as well as risk of damage to neurovascular structures. Therefore, minimally invasive fixation is increasingly being used as an alternative to external fixators for the treatment of anterior pelvic ring fractures [6, 7]. Insertion of supraacetabular pedicle screws connected via a subcutaneous contoured rod tunnelled just below the belly crease (so-called bikini area) [8], which is known as the subcutaneous anterior pelvic fixation (INFIX) technique, has the advantages of convenience, minimal invasiveness and blood loss, and less discomfort for patients with anterior pelvic ring injury $[9,10]$. However, there is limited evidence for the efficacy of INFIX and many aspects require improvement such as the persistence of pubic pain, soft tissue irritation, loss of reduction, and especially fixator loosening $[5,11]$. The most common complication reported was 
lateral femoral cutaneous nerve palsy (occurred temporarily in 0-30\%), and heterotopic ossification(H0) (reported in 0-25\%)

We previously showed that modified pedicle screw-rod fixation (MPSRF) can lead to more rapid recovery from anterior pelvic ring fractures [12]. However, it is unclear how it compares to INFIX in terms of strength and stability. We hypothesized that the MPRSF could bring sufficient biomechanical stability compare with INFIX. In order to demonstrate this hypothesis, We examined the postoperative biomechanical characteristics of the implants when patients assumed single-/dual-leg standing and sitting postures by finite element analysis (FEA), a computational method for assessing and predicting the outcome of surgery that eliminates variations in bone quality, fracture pattern, bone anatomy, and fixation location upon application of physiological loads that has been used to analyse and predict the outcome of surgery. [13-15]

\section{Methods}

\section{Patients and methods}

This retrospective study was reviewed and approved by the Ethics Committee of Zhongshan Hospital. All procedures were performed in accordance with the Declaration of Helsinki and strictly adhered to institutional guidelines. A total of 63 patients with anterior pelvic ring injury were enrolled from January 2014 to January 2017, with a minimum follow-up of 13 months. Inclusion criteria were unstable anterior pelvic ring fracture with a stable posterior ring (either intact or recovered after fixation) diagnosed by the senior trauma surgeon, hemodynamic stability, and full consciousness. Patients with an open contaminated wound, who were lost to follow-up before 3 months, had a pathologic fracture, or were $<16$ years of age were excluded (Fig. 1).

The pelvis of each patient was examined by preoperative radiography (including anterior-posterior [AP], inlet, and outlet views) and computed tomography (CT). Imaging data were analysed by 2 senior orthopedists according to the Arbeitsgemeinschaft für Osteosynthesefragen (AO) and Orthopaedic Trauma Association (OTA) modified tile type classification. Post-surgery clinical outcome was assessed based on Tornetta and Matta grade, Majeed score, and complications.

\section{Surgical technique}

If required, surgical reduction of the posterior pelvic ring injury was performed prior to fixation as previously described [12]. For MPSRF, a 3- to 4-cm oblique incision was made over each anterior inferior iliac spine (AIIS). A bony tunnel from the AllS to the posterior superior iliac spine was created with a pedicle finder. A polyaxial pedicle screw with a diameter of $7 \mathrm{~mm}$ and length of $60 \mathrm{~mm}$ was inserted into the tunnel to a depth of about $2 \mathrm{~cm}$ from the bone surface to avoid compression of vascular tissue. A subcutaneous tunnel was created from the incisions at bilateral AlIS to the Pfannenstiel incision over the deep fascia. The curved titanium rod was inserted to connect the 3 bilateral pedicle screws via the subcutaneous tunnel. After confirming that there was sufficient space between the rod and bone by 
fluoroscopy, the screws were tightened with a torque screwdriver, with those at bilateral AllS tightened before the one at the pubic tubercle. A representative case is shown in Supplementary Figure 1 (S1).

The INFIX was inserted in the same manner. Briefly, 2 polyaxial pedicle screws were placed at bilateral AllS. A subcutaneous tunnel was created from 1 side of the AllS to the other, and the precontoured titanium rod was connected to the 2 screws via the tunnel. Fracture reduction was performed by the same method as described above. A representative case is shown in Supplementary Figure 2 (S2).

\section{Postoperative management and follow-up}

Functional exercises of the lower limbs and joints were initiated in bed as early as possible after the operation to prevent deep vein thrombosis; regular wound and dressing care was performed in the outpatient clinic until sutures were removed at 2 weeks' post-surgery. Crutch-assisted partial weight bearing was permitted at 6 and 10 weeks for AO/OTA type B and C injuries, respectively, as long as the pain was tolerable. Full weight bearing was allowed if osseous union was confirmed by radiography. Physical therapy was prescribed for muscle strengthening and gait training. Hardware removal was performed between 12 and 14 months after the surgery.

Patients were followed up by phone or at the clinic. Routine follow-ups were scheduled for postoperative weeks 4 and 8 and months $6,12,18$, and 24. Radiographic images included a 3-view (AP, inlet, and outlet) pelvis series and all patients were asked about pain, numbness, and motor ability at each follow-up. Physical and neurologic examinations were performed to evaluate irritation around the implant as well as the condition of the lateral femoral cutaneous nerve (LFCN) and femoral nerves.

\section{Radiographic and functional outcome assessment}

The extent of fracture reduction was evaluated by postoperative $X$-ray examination and graded according to the method of Tornetta and Matta [16] as excellent (displacement $\leq 4 \mathrm{~mm}$ ), good (5-10 mm), fair (10$20 \mathrm{~mm}$ ), or poor (displacement $>20 \mathrm{~mm}$ ). A grade of excellent or good was regarded as a satisfactory outcome.

Clinical outcome measures included operation time, hospital stay, intraoperative blood loss, and postoperative complications. The Majeed rating system was used to assess functional outcome at 6 months, the time of implant removal (10-14 months), and last follow-up; the variables were pain (30 points), standing (36 points), sitting (10 points), sexual intercourse (4 points), and work (20 points). Aggregate scores were classified as excellent (>85), good (70-84), fair (55-69), or poor (<55) [17].

\section{FEA}

In order to define the solid geometry of the pelvis, we constructed a model of the pelvis of a healthy 32year-old male (height, $175 \mathrm{~cm}$ and weight, $73 \mathrm{~kg}$ ) based on images from a CT scan. The material properties of the model were obtained from previous studies (Table 3) [1]. A 2-cm gap was created at the right superior and inferior rami to simulate injury using Geomagic Studio software (3D Systems Inc, Rock 
Hill, SC, USA). 3-Dimensional models of the rod and screws were constructed using Creo v3.0 software (Parametric Technology Corp, Needham, MA, USA). The materials for the different models and implants were assumed to be elastic, isotropic, and linear. A value of 0.33 was set as Poisson's ratio (y) for both cortical and cancellous bone.

Models of the intact pelvis and injured pelvis with the 2 types of fixation were imported into Workbench v.17.0 software (ANSYS Inc, Canonsburg, PA, USA) to analyse equivalent von Mises (VM) stress and displacement. We performed convergent analysis to balance the accuracy and efficiency of the finite element simulation by adjusting element size. Interaction surfaces including sacrum, sacroiliac cartilage ilium, pubic rami, and bone implant were fully constrained and a vertical load of $600 \mathrm{~N}$ was imposed on the superior surface of sacrum to simulate upper body weight. The unilateral (left/right) acetabulum, bilateral acetabulum, and bilateral ischium were fully constrained to simulate single-leg (left/right) and dual-leg standing and sitting postures, respectively (Supplementary Figure 3).

\section{Statistical analysis}

Data were analysed using SPSS v20.0 software (SPSS Inc, Chicago, IL, USA). Data satisfying the conditions of normality are presented as means \pm standard deviation; non-normal data are presented as medians and quartiles. Differences in categorical variables (e.g. postoperative complications) were assessed with the chi-squared test or Fisher's exact test, whereas differences in continuous variables were evaluated with the Student's t test when the assumptions of normality were valid. A $P$ value $<0.05$ was considered significant.

\section{Results}

\section{Clinical outcome}

A consecutive series of 63 patients with type $B$ and type $C$ fractures were enrolled. The causes of injury were traffic accident $(n=25)$, fall $(n=21)$, and other $(n=14)$. Average age and sex ratio did not differ between the 2 groups. The mean injury severity score of patients who underwent INFIX was 25 points (range, 19-29 points); that of patients who underwent MPSRF was 22 points (range, 19-29 points). The 2 groups were similar with respect to OTA classification and causes of injury as well as time to pelvic surgery. However, the INFIX group had longer operation time and greater blood loss than the MPSRF group. There was also a statistically significant difference between the 2 groups in terms of hospital stay (Table 1).

The median follow-up was 20 months (range, 13-27 months) in the INFIX group and 22 months (range, 17-30) in the MPSRF group. All patients' fractures healed without incident after the operation. For Tornetta and Matta grade, the MPSRF group had a higher satisfactory rate (["Excellent" + "Good"] / total number of patients) compared to the INFIX group (73.33\% vs $63.63 \%)$, but the difference was not statistically significant $(P=0.409)$. The MPSRF group had a higher Majeed score than the INFIX group at 6 
months post-surgery $(P<0.001)$ and at the time of implant removal $(P=0.012)$. However, there was no difference in the scores of the two groups at 22 months (Table 2).

\section{Complications}

LFCN irritation was observed in 10 patients (33.3\%) in the MPSRF group and 8 patients $(24.2 \%)$ in the INFIX group. The symptoms mainly manifested as anterolateral skin numbness of the affected thigh. In most cases this was alleviated by physical therapy at postoperative month 6 , and nearly two-thirds of the 26 patients recovered after implant removal. There was 1 case of unilateral femoral nerve palsy immediately after the operation in the MPSRF group, and 1 patient complained of tardive impingement pain without motor dysfunction at 3 months' post-surgery in the INFIX group. Additionally, 3 patients who underwent INFIX experienced implant loosening (Table 2).

\section{Biomechanical characteristics of intact model}

We constructed, meshed, and solved models of an intact pelvis and injured pelvises with 2 types of fixation in single- (right/left) and dual-leg standing and sitting postures. Displacement distribution and VM stress of the intact pelvis were bilaterally symmetric and cantered on the sacrum in the dual-leg standing and sitting postures. The maximum stress was $28,30,21$, and $18, \mathrm{MPa}$ on the right face of the sacrum in the single-leg (left/right) and dual-leg standing and sitting postures, respectively (S1 B).

\section{Evaluation of fixation stability in the injury model}

A lower maximum displacement of the pelvis in the model indicates higher stability or fixation stability. For the finite element models in the 4 postures, the injury model treated by MPSRF had lower maximum pelvis displacement than that treated by INFIX, especially in the sitting posture $(0.37 \mathrm{vs} 0.21 \mathrm{~mm})$ and single-leg standing posture (left, 0.51 vs $0.38 \mathrm{~mm}$ and right, $0.56 \mathrm{vs} 0.42 \mathrm{~mm}$ ), indicating that MPSRF had greater stability (Supplementary Figure 4). Moreover, maximum pelvis displacement in the injury model treated by MPSRF was comparable to that of the intact pelvis. Maximum VM stress was slightly higher in the injury model treated by INFIX on the face of the sacrum compared to the MPSRF-treated and intact models, with a maximum VM stress in the single-leg (left) posture of $57.3 \mathrm{MPa}$ vs 21 and $28 \mathrm{MPa}$, respectively (Figure 2 and 3 ), which was consistent with the observed trend in maximum pelvis displacement. Thus, the injured pelvis showed greater stiffness when treated by MPRSF compared to INFIX.

\section{Risk of implant failure and micromotion in the injury model}

A lower maximum implant displacement indicates a lower risk of implant failure and micromotion and higher fixation stability. For the finite element models in the 4 postures, the injury model treated by MPSRF had $30 \%$ and $36 \%$ lower maximum displacement compared to the INFIX-treated model in the standing posture ( 0.40 vs $0.28 \mathrm{~mm}$ ) and sitting posture $(0.30$ vs $0.19 \mathrm{~mm})$, respectively, indicating a lower risk of implant failure and micromotion (Supplementary Figure 4). 
A lower maximum VM stress represents a lower risk of pelvic breakage. Maximum implant stress was slightly higher in the injury model treated by MPSRF compared to INFIX in all 4 postures (Supplementary Fig. S4). However, maximum VM stress occurred at the central region of the third pedicle screw of the pubis in the model treated by MPSRF in the standing and sitting postures (Figs. 2D and 3D), implying a lower risk of micromotion. In the single-leg (left/right) posture, the maximum VM stress was on the side opposite to the implant in the INFIX model but on the same side in the MPSRF model (Figs. 2D and 3D). Taken together, the FEA results indicate that anterior pelvic ring fractures treated by MPRSF are stiffer, have greater fixation stability, and lower risk of implant failure than those treated by INFIX.

\section{Discussion}

Unstable pelvic ring injuries typically result from high-energy trauma and are considered by orthopaedic surgeons as one of the most clinically challenging lesions. Open reduction and internal fixation is the standard operative treatment for hemodinamically stable patients with anterior pelvic ring injuries. Nevertheless, the major issue concerning this method is poor patient tolerance leading to frequent complaints when patients present decubitus, pin tract infection as well as cannot sitting. To address these drawbacks, Minimally invasive fixation of pelvic fractures as an adjunct to posterior fixation is becoming more common and can effectively improve anterior pelvic stability and restore function in the pelvis, while being associated with minimal traumatic invasion and fewer complications [18]. INFIX is a technique in which pedicle screws are placed on the supra-acetabular corridor with a crossrod in the bikini area [19]. INFIX has recently emerged as an alternative to traditional surgical methods with the advantages of few soft tissue complications, improved biomechanical construction, and no disruption of sitting posture or screwdriver function. MPRSF combines the advantages of INFIX and pelvic bridging. The safety and effectiveness of minimally invasive fixation of pelvic fractures have been demonstrated by several anatomic and clinical studies, although fixation stability remains a concern $[20,21]$. Additionally, few studies have investigated whether these two fixation techniques lead to sufficiently strong implants and adequate biomechanical reduction.

The FEA results showed that displacement distribution and VM stress were similar in the injured pelvis models treated by MPRSF and INFIX compared to the intact pelvis, indicating that both fixation techniques can effectively treat anterior pelvic ring fractures. However, maximum displacements of the pelvis and implant were lower in the MPRSF group than in the INFIX group in the single- (left/right) and dual-leg standing and sitting postures, indicating greater stability. On the other hand, the injury model treated by MPSRF showed slightly higher maximum implant stress compared to the INFIX group in all four models, which may be attributable to part of the stress being concentrated on the third screw and obstructing implant micromovement.

Although the MPRSF group had a longer operation time and greater intraoperative blood loss, there were no significant differences in hospital stay and postoperative complications between the 2 groups. Thus, MPRSF only expanded the local skin incision without causing permanent neurovascular injury and did not increase the risk of postoperative complications. However, radiographic (Tornetta and Matta grade) 
and functional (Majeed score) outcomes of the MPRSF group were improved compared to the INFIX group, especially at 6 months after surgery. Thus, while both fixation methods can restore stability to the anterior pelvic ring and pelvic function, MPRSF promotes the latter at an earlier time point following operation.

LFCN irritation is the most common postoperative iatrogenic complication [22]. In a multicentre review, $30 \%(21 / 91)$ of patients had LFCN irritation although in most cases it was self-limiting and improved once the implant was removed [5]. In line with this study, in our investigation the rate of LFCN injury was $33.3 \%$ (10/30) in the MPRSF group and $24.2 \%(4 / 33)$ in the INFIX group. A case series of LFCN irritation suggested that screws that are too deeply or insufficiently embedded in the bone and inadequate prebending of the rod can lead to irritation of the LFCN and sartorius muscle. To prevent this, a rod-to-bone distance of $20-25 \mathrm{~mm}$ (30-40 $\mathrm{mm}$ for obese patients) but $<40 \mathrm{~mm}$ is recommended [23]. We first locked the screws at bilateral AllS so that the pullout strength of the screw was mainly concentrated in the supraacetabular region, where bone density is high. The screw at the pubic tubercle-which has relatively sparse bone-was then locked, thus providing auxiliary support. Furthermore, additional screws should not be placed too close to the lateral pubis to avoid damaging the spermatic cord or round ligament. For unilateral pubic rami fractures, the screw was fixed into the fracture side if the fracture line was far away from the pubic symphysis; otherwise, it was inserted on the uninjured side. For bilateral pubic rami fractures, the screw was fixed into the side with less injury.

Recent studies using the INFIX or MPRSF technique have reported potentially devastating complications, especially femoral nerve palsy. There was one such case in the MPRSF group, but symptoms gradually disappeared once emergency screw adjustment was performed, and there was no permanent nerve damage after implant removal. In a case series of iatrogenic femoral nerve palsy, it was suggested that femoral nerve compression occurs as a result of impingement of the implant on the psoas sheath; meanwhile, delayed palsy may be caused by engorgement of the psoas with blood and/or a change in pressure [24]. The authors noted that this could be avoided by placing the interconnecting rod in such a way that it does not limit the space for the psoas and femoral nerve. Our solution for reducing neurovascular compression was to bend the connecting rod outward in the horizontal direction (Figure 4). Although follow-up is ongoing, the patient's neurovascular compression symptoms have been significantly alleviated.

Despite the positive clinical outcomes and FEA results, our study had several limitations. Firstly, this was a single-centre retrospective study with a relatively small sample size; more cases should be examined in a multicentre investigation, with long-term functional assessment. Secondly, the characteristics of the implant materials are not consistently reported; as such, our results depend on input parameters. Additionally, the FEA model was patient-specific and ignored the effects of ligaments and muscles on the stability of the pelvis and implant devices. Finally, the angle of applied force was constant although variations in the angle may have influenced VM stress and displacement, as reported in a previous study [25]. 


\section{Conclusion}

In conclusion, our study demonstrates that both MPRSF and INFIX used as a minimally invasive procedure in the treatment of unstable pelvic anterior ring fracture provides adequate biomechanical stability. However, fixation stability was greater with MPRSF than with INFIX, which was associated with a lower risk of implant failure according to the results of FEA. Combined with the superior clinical and radiologic outcomes associated with external fixation, MPRSF is the preferred option for the treatment of unstable pelvic anterior ring fractures.

\section{Declarations}

All procedures were performed in accordance with the Declaration of Helsinki and strictly adhered to institutional guidelines.

\section{Availability of data and materials}

The datasets used and/or analysed during the current study are available from the corresponding author on reasonable request.

\section{Competing interests}

The authors declare that they have no competing interests.

\section{Funding}

This study was supported by the Project of Qingpu District Science and Technology Commission (QKY2018-11) and the Project of Qingpu Branch of Zhongshan Hospital (QYZ2019-02 and QY2019-04).

\section{Authors' contributions}

$\mathrm{ZR}$, SD, and FC conceived and devised the study. SD, FC, and JH performed the study. JH, MG, FW, and JL analyzed the data. SD, ZR and FC wrote the paper. ZR and XW revised the manuscript. ZR obtained the funding and supervised the whole project. All authors have contributed to the final version and approved the publication of the final manuscript.

\section{Acknowledgments}

Not applicable.

\section{Abbreviations}

AllS, anterior inferior iliac spine; AO, Arbeitsgemeinschaft für Osteosynthesefragen; AP, anterior-posterior; $\mathrm{CT}$, computed tomography; FEA, finite element analysis; INFIX, anterior subcutaneous internal pelvic 
fixation; LFCN, lateral femoral cutaneous nerve; MPSRF, modified pedicle screw-rod fixation; OTA, Orthopaedic Trauma Association; VM, von Mises.

\section{References}

1. Ricci PL, Maas S, Kelm J, Gerich T. Finite element analysis of the pelvis including gait muscle forces: an investigation into the effect of rami fractures on load transmission. J Exp Orthop. 2018; 5:33.

2. Chen W, Lv H, Liu S, Liu B, Zhu Y, Chen X, et al. National incidence of traumatic fractures in China: $A$ retrospective survey of 512187 individuals. Lancet Glob Health. 2017;5: e807-17.

3. Tile M. Management of unstable injuries of the pelvic ring. J Bone Joint Surg Br. 1999; 81:941-3.

4. Gardner MJ, Nork SE. Stabilization of unstable pelvic fractures with supraacetabular compression external fixation. J Orthop Trauma. 2007; 21:269-73.

5. Vaidya R, Kubiak EN, Bergin PF, Dombroski DG, Critchlow RJ, Sethi A, et al. Complications of anterior subcutaneous internal fixation for unstable pelvis fractures: A multicenter study. Clin Orthop Relat Res. 2012; 470:2124-31.

6. Osterhoff G, Tiziani S, Ferguson SJ, Spreiter G, Scheyerer MJ, Spinas GL, et al. Mechanical testing of a device for subcutaneous internal anterior pelvic ring fixation versus external pelvic ring fixation. BMC Musculoskelet Disord. 2014; 15:111.

7. Gardner MJ, Mehta S, Mirza A, Ricci WM. Anterior pelvic reduction and fixation using a subcutaneous internal fixator. J Orthop Trauma. 2012; 26:314-21.

8. Vaidya R, Colen R, Vigdorchik J, Tonnos F, Sethi A. Treatment of unstable pelvic ring injuries with an internal anterior fixator and posterior fixation: Initial clinical series. J Orthop Trauma. 2012; 26:1-8.

9. Cole PA, Gauger EM, Anavian J, Ly TV, Morgan RA, Heddings AA. Anterior pelvic external fixator versus subcutaneous internal fixator in the treatment of anterior ring pelvic fractures. $J$ Orthop Trauma. 2012;26: 269-77.

10. Hiesterman TG, Hill BW, Cole PA. Surgical technique: A percutaneous method of subcutaneous fixation for the anterior pelvic ring: the pelvic bridge. Clin Orthop Relat Res. 2012;470: 2116-23.

11. Owen MT, Tinkler B, Stewart R. Failure and salvage of "INFIX" instrumentation for pelvic ring disruption in a morbidly obese patient. J Orthop Trauma. 2013; 27:e243-6.

12. Ding S, He X, Zhang G, Ruan Z, Wu X. Modified internal fixator for anterior pelvic ring fractures versus conventional two-screw fixation. Eur J Trauma Emerg Surg. 2019 May 30. doi: 10.1007/s00068-01901164-8

13. Ramaswamy R, Evans S, Kosashvili Y. Holding power of variable pitch screws in osteoporotic, osteopenic and normal bone: Are all screws created equal? Injury. 2010; 41:179-83.

14. Seyed Vosoughi A, Joukar A, Kiapour A, Parajuli D, Agarwal AK, Goel VK, et al. Optimal satellite rod constructs to mitigate rod failure following pedicle subtraction osteotomy (PSO): A finite element study. Spine J. 2019; 19:931-41. 
15. Joukar A, Shah A, Kiapour A, Vosoughi AS, Duhon B, Agarwal AK, et al. Sex specific sacroiliac joint biomechanics during standing upright: A finite element study. Spine (Phila Pa 1976). 2018;43: E1053-60.

16. Tornetta P, Matta JM. Outcome of operatively treated unstable posterior pelvic ring disruptions. Clin Orthop Relat Res. 1996; Aug:186-93.

17. Majeed SA. Grading the outcome of pelvic fractures. J Bone Joint Surg Br. 1989; 71:304-6.

18. Cole PA, Dyskin EA, Gilbertson JA. Minimally-invasive fixation for anterior pelvic ring disruptions. Injury. 2015;46: S27-34.

19. Kuttner M, Klaiber A, Lorenz T, Füchtmeier B, Neugebauer R. [The pelvic subcutaneous cross-over internal fixator]. Unfallchirurg. 2009; 112:661-9 [article in German].

20. Becker CA, Kammerlander C, Kussmaul AC, Woiczinski M, Thorwachter C, Zeckey C, et al. Modified less invasive anterior subcutaneous fixator for unstable Tile-C-pelvic ring fractures: A biomechanical study. Biomed Eng Online. 2019; 18:38.

21. Reichel LM, MacCormick LM, Dugarte AJ, Rizkala AR, Graves SC, Cole PA. Minimally invasive anterior pelvic internal fixation: An anatomic study comparing Pelvic Bridge to INFIX. Injury. 2018; 49:309-14.

22. Fang $\mathrm{C}$, Alabdulrahman $\mathrm{H}$, Pape HC. Complications after percutaneous internal fixator for anterior pelvic ring injuries. Int Orthop. 2017; 41:1785-90.

23. Scherer J, Tiziani S, Sprengel K, Pape HC, Osterhoff G. Subcutaneous internal anterior fixation of pelvis fractures - which configuration of the InFix is clinically optimal? -A retrospective study. Int Orthop. 2018; 43:2161-2166.

24. Chaus GW, Weaver MJ. Anterior subcutaneous internal fixation of the pelvis: Placement of the INFIX. Oper Tech Orthop. 2015; 25:262-9.

25. Grassi L, Schileo E, Taddei F, Zani L, Juszczyk M, Cristofolini L, et al. Accuracy of finite element predictions in sideways load configurations for the proximal human femur. J Biomech. 2012; 45:394-9.

\section{Tables}

Table 1. The demographics of two groups (ISS, injury severity score). 


\begin{tabular}{lccc}
\hline Parameter & MPRSF group(n=30) & INFIX group(n=33) & $P$ value \\
\hline Ageđyears $\square$ & $34.4 \pm 17.5$ & $36.2 \pm 16.9$ & $0.856^{\mathrm{a}}$ \\
Gender (male/female) & $16 / 14$ & $20 / 13$ & $0.560^{\mathrm{b} 1}$ \\
ISS & $22(19,29)$ & $25(21,32)$ & $0.283^{\mathrm{a}}$ \\
AO/OTA/modified Tile type & $4 / 15 / 6$ & $7 / 17 / 3$ & $0.136^{\mathrm{b} 2}$ \\
B1/B2/B3 & $2 / 3 / 0$ & $4 / 2 / 0$ & $0.846^{\mathrm{b} 1}$ \\
C1/C2/C3 & 13 & 15 & $0.782^{\mathrm{d}}$ \\
Injury mechanism & 11 & 10 & $0.015^{\mathrm{a}}$ \\
Traffic injury & 6 & 8 & $0.036^{\mathrm{a}}$ \\
Falling injury & $5(0,14)$ & $6(0,16)$ & $0.028^{\mathrm{a}}$ \\
Other & $77.3 \pm 11.2$ & $52.6 \pm 12.9$ & \\
Time to surgery(d) & $103.6 \pm 10.2$ & $87.7 \pm 9.3$ & \\
Operation time(min) & $11(7,18)$ & $14(8,21)$ & \\
Intraoperative blood loss (ml) & & & \\
Hospital stays (days) & &
\end{tabular}

a: Two-sample t-test. b1: Pearson Chi-squared Test. b2: Cochran-Mantel-Haenszel Test.

c: Fisher's Exact Test d: Log Rank Test

Table 2. Postoperative functional outcome grading and complications.

Modified group Conventional group $P$ value

$(n=30) \quad(n=33)$

\begin{tabular}{lccc}
\hline Tornetta and Matta grading & 12 & 6 & $0.037^{\mathrm{b} 2}$ \\
Excellent & 13 & 15 & $0.409^{\mathrm{b} 1}$ \\
Good & 4 & 9 & \\
Fair & 1 & 3 & \\
Poor & $22 / 30,(73.33 \%)$ & $21 / 33,(63.63 \%)$ & \\
Satisfactory rate & & & \\
& & & \\
The Majeed score & $81.5 \pm 10.4$ & $76.3 \pm 11.2$ & $<0.001^{\mathrm{a}}$ \\
Postoperatively 6 months & $84.8 \pm 8.63$ & $80.7 \pm 7.37$ & $0.012^{\mathrm{a}}$ \\
The time of implant removal & $88.4 \pm 7.12$ & $87.6 \pm 6.45$ & $0.065^{\mathrm{a}}$ \\
The last follow up(22 months) & 12 & 14 & $0.307^{\mathrm{c}}$ \\
Postoperative complications (n) & 10 & 8 & \\
LFCN irritation (n) & 1 & 0 & \\
Femoral nerve palsy (n) & 0 & 1 & \\
Tardive impingement pain (n) & 1 & 2 & \\
Infection & 0 & 3 & \\
Implant loosening & & \multicolumn{2}{c}{} \\
\hline
\end{tabular}

a: Student t-test. b1: Pearson Chi-squared test. b2: Cochran-Mantel-Haenszel Test.

c: Fisher's Exact Test.

Table 3. Material properties used in the finite element models.

\begin{tabular}{lcc}
\hline Material & Elastic Modulus(MPa) & Poisson Ratio $(\mu)$ \\
\hline Sacrum cortical bone & 6200 & 0.33 \\
Ilium cortical bone & 17000 & 0.33 \\
Sacrum cancellous bone & 1400 & 0.30 \\
Ilium Cancellous bone & 130 & 0.20 \\
Screws/rod & 110000 & 0.30 \\
Cartilage & 60 & 0.30 \\
\hline
\end{tabular}




\section{Enrolment}

Patient screened for eligibility

$(n=237)$

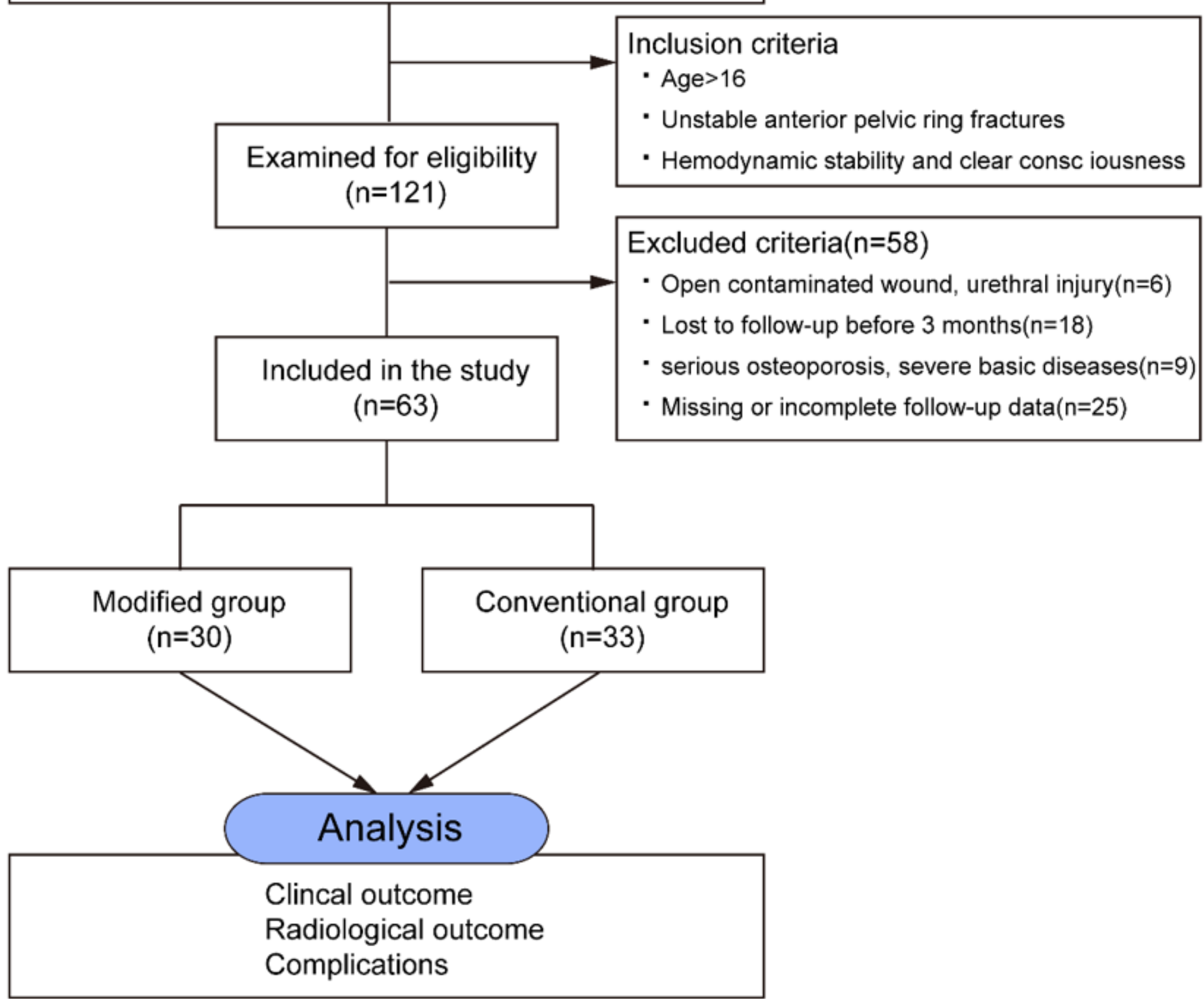

Figure 1

Flow diagram of patient's exclusion. Flow diagram of patient's exclusion. 63 patients with anterior pelvic ring injuries were enrolled from January 2014 to January 2017 in our institution. 


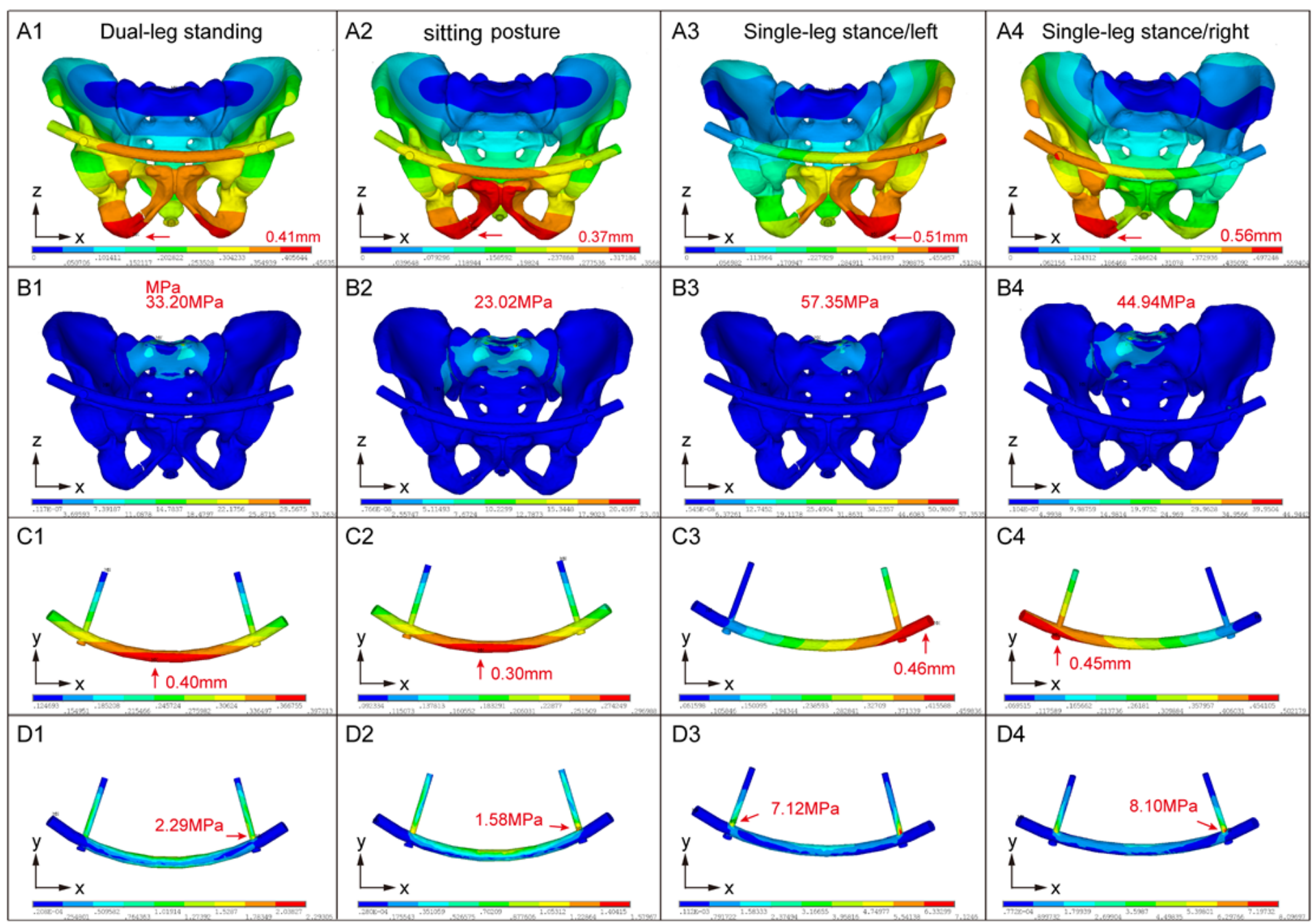

Figure 2

FE injured model using INFIX under four posture. including the dual-leg standing, siting posture and single-leg stance(left/right). A: The displacement of the injured pelvis with INFIX. B: The Von Mises stresses applied to the injured pelvis with INFIX. C: The displacement of the implant device. D: The Von Mises stresses applied to the implant device. 


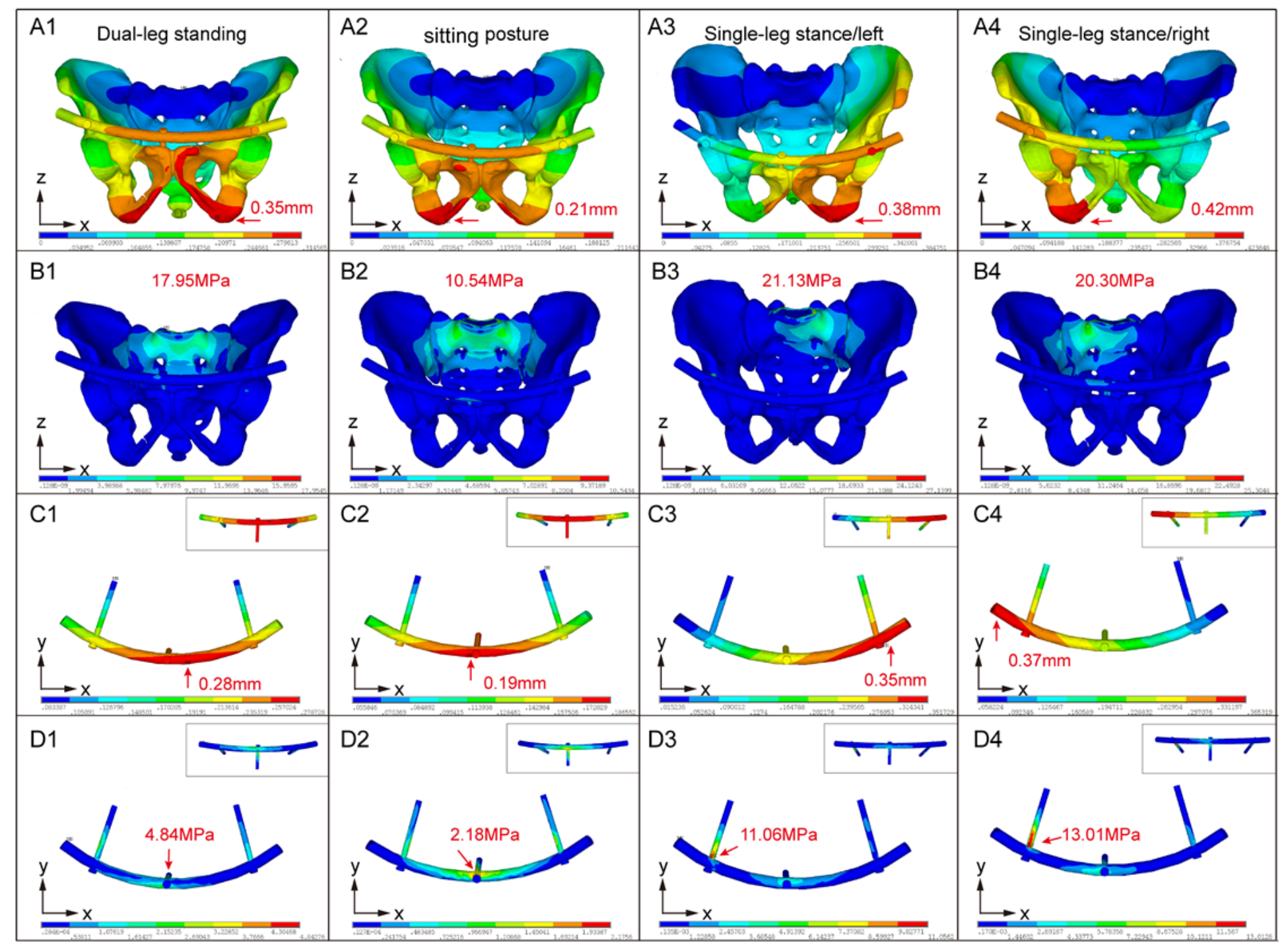

Figure 3

FE injured model with MPSRF under four posture. including the dual-leg standing, siting posture and single-leg stance(left/right). A: The displacement of the injured pelvis with MPSRF. B: The Von Mises stresses applied to the injured pelvis with MPSRF. C: The displacement of the implant device. D: The Von Mises stresses applied to the implant device. 

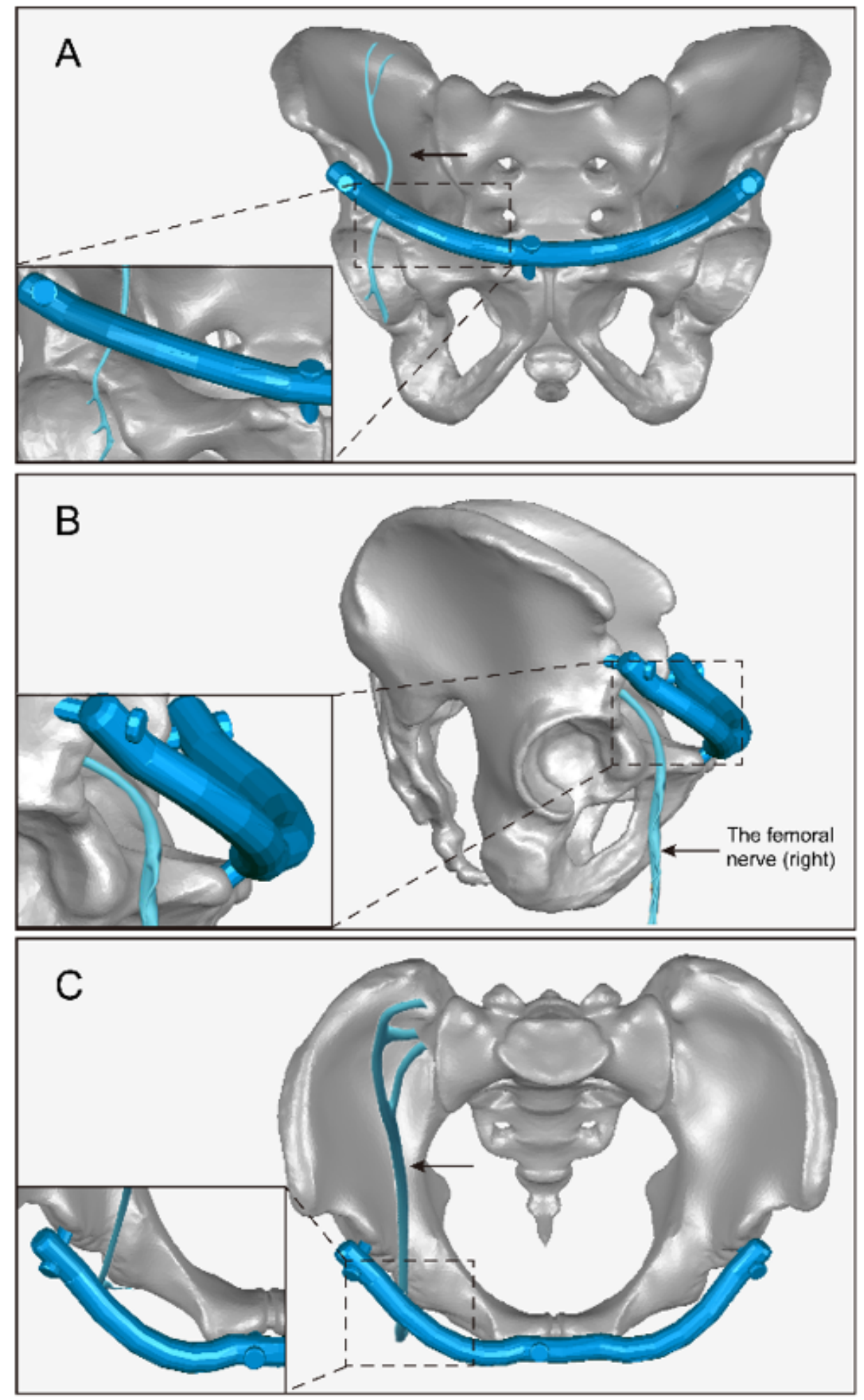

\section{Figure 4}

The diagram of the improvement applied to the MPSRF. The diagram of the improvement applied to the MPSRF, bending the connecting rod outward in the horizontal direction.

\section{Supplementary Files}


This is a list of supplementary files associated with this preprint. Click to download.

- Supplement.docx 